\title{
Tele-Healthcare Monitoring System-Based for the Management of Diabetes Emergencies
}

\author{
Julius Olasunmibo Ogunniyi ${ }^{1}$, Justice Ono Emuoyibofarhe ${ }^{2}$, Michael Adeyemi Olamoyegun ${ }^{3 *}$ \\ ${ }^{1}$ Department of Computer Engineering, Faculty of Engineering, Elizade University, Ilara-Mokin, Nigeria \\ ${ }^{2}$ Department of Information System, Faculty of Computing and Informatics, Ladoke Akintola University of Technology, \\ Ogbomoso, Nigeria \\ ${ }^{3}$ Department of Medicine, Endocrinology, Diabetes \& Metabolism Unit, Faculty of Clinical Sciences, Ladoke Akintola University \\ of Technology/LAUTECH Teaching Hospital, Ogbomoso, Nigeria \\ Email: *maolamoyegun@lautech.edu.ng
}

How to cite this paper: Ogunniyi, J.O., Emuoyibofarhe, J.O. and Olamoyegun, M.A. (2021) Tele-Healthcare Monitoring System-Based for the Management of Diabetes Emergencies. E-Health Telecommunication Systems and Networks, 10, 83-94. https://doi.org/10.4236/etsn.2021.104005

Received: August 7, 2021

Accepted: September 22, 2021

Published: September 25, 2021

Copyright $\odot 2021$ by author(s) and Scientific Research Publishing Inc. This work is licensed under the Creative Commons Attribution International License (CC BY 4.0).

http://creativecommons.org/licenses/by/4.0/

cc) (i) Open Access

\begin{abstract}
Introduction: Diabetes mellitus is a global health burden whose prevalence is increasing worldwide. Remote patient monitoring, an automated process which involve transmission of data swiftly from the person with diabetes to the health care providers is often considered to be a technological solution to the challenges of health care delivery with potential of reducing both morbidity and mortality. While the use of telehealth in diabetes management is becoming more prominent, however its use in the management of diabetes emergencies is not very common. Objective: To evaluate telehealth intervention in the management of patient with diabetes in the emergency setting with resultant early diagnosis and management. Methods: This paper presents a design, implementation and evaluation of Tele-healthcare monitoring system with the aids of GPS, smartphone and cellular network infrastructure to monitor patients with diabetes especially during diabetic emergencies. This system depends on remote monitoring of patient when away from the place of domicile or their treating doctor or family members using patient's data. Patient can also be linked to nearby hospitals using GPS and the attending doctors would have access to patient's data. Alarm is also issued by the system to the next-of-kin/family members or care giver by sending emergency SMS including the location, time and patient's clinical condition. The developed system consists of mobile application and web based application. The Mobile application was developed with JAVA programming language and SQLite database. Results: The result from the evaluation of the developed system shows effectiveness of $83.33 \%$ and mean users' perception of $94 \%$. Conclusion: This system is simple and cost-effective to be implemented and enables early intervention when the blood glucose is abnormally high or low in order to save life of the patient without delay.
\end{abstract}




\section{Keywords}

Diabetes, Telehealth, Remote Monitoring, Smartphone, GPS

\section{Introduction}

Diabetes is a chronic metabolic disorder of carbohydrates, protein and fats characterized by hyperglycaemia (elevated blood glucose) due to absolute or relative insulin secretion or actions or both [1]. Globally the prevalence of diabetes was estimated to be 463 million in 2019 and has been projected to reach 578 million in 2030 and 700 million by 2045 [2]. Most increases in the prevalence of diabetes occur in low- and middle-income countries of the world including Nigeria which has the largest number of people living with diabetes in the sub-Saharan region [2]. In Nigeria diabetes is evidently becoming a national problem and the difficulties of adequately treating all groups within the population are well known. Due to the limited number of diabetes specialists required in the management of diabetes and its myriad complications, many of the individuals with diabetes who developed complications are not adequately managed especially those who reside in rural or remote places [3].

Advances in digital technology and especially mobile smartphone technology have led to a plethora of innovative strategies aiming to improve the self-management skills of patients with chronic diseases and especially diabetes. Telemedicine is been defined as the use of telecommunications to support healthcare. It includes timely transmission and remote interpretation of patient data for early interventions and management of patients [4]. Telemedicine interventions can be communicated from handheld hardware devices to remote Web server. Hardware for transmission may include mobile phones, handheld personal digital assistant devices, and portable/laptop computers [5] [6]. Data may be transmitted in the form of voice messages over the phone, text messages, email messages and live streaming audio/video over the internet. The advent of telehealth has the potential to fit and care for patients where it is needed. Telehealth can assist to monitor conditions remotely and it can help intervene when acute or emergency conditions arise. Specifically this type of telehealth would help diabetic patients at school, elderly home and when journeyed away from area of domicile or away from hospital of care. Indeed, mobile health enables the use of telecommunication technologies by health care providers and patients to exchange information and health services for patient care and monitoring.

Diabetes is associated with increasing morbidity and mortality especially in developing countries mainly due to its acute complications especially diabetes ketoacidosis (DKA), hyperglycaemic hyperosmolar state (HHS), [both of which are characterised by marked elevated blood glucose] and hypoglycaemia (low blood glucose). These acute complications of diabetes usually present as an emergency and both are among the common causes of morbidity with mortality 
in Nigeria ranging from 10\% - 15\% [7] [8]. In the United States, diabetic ketoacidosis (DKA) accounts for more than 110,000 hospitalizations annually, with mortality ranging from $2 \%$ to $10 \%$ [8] [9] [10] [11]. Hyperglycemic hyperosmolar state (HHS) is much less common but confers a much greater mortality [9].

Many measures are currently in use to prevent or rather detect early occurrence or identify diabetic patients who develop these acute diabetic complications. For instance, as many as people with diabetes know, carrying Emergency Medical Identification (EMI) is an important part of daily life. Currently, EMI is commonly found in the form of wearable jewellery like necklace, hand bands with diabetes inscriptions, and wallet cards. Some people with diabetes even choose EMI with body tattoo. However, it is not something anyone ever wants to have to use due to fear of stigmatization especially in developing countries like Nigeria. Mobile devices like smartphones offer a unique solution to the EMI problem because they are already routinely carried by a large portion of society. Hence, this eliminates stigmatization associated with EMI and provides privacy to the users as there would be no visible information on a persons' medical condition unless an emergency requires accessing the EMI, compared with medical alert jewellery.

Remote healthcare is an emerging research field as the world moves towards remote monitoring, real-time and fast detection of illnesses. Remote healthcare has many categories, (e.g. telehealth, mobile health) all of which mean monitoring of patients outside hospital conditions by the means of technology. The advantages of remote monitoring of patients are: early and real-time detection of illnesses, ability to continuously monitor patients, prevention of worsening of illnesses and untimely deaths, cost reduction in hospitalizations, reduce the frequency of hospitalizations, obtain more accurate readings while permitting usual daily activities for patients, improve efficiency in healthcare services by utilizing communication technology, emergency medical care, service for patients with mobility issues, and emergency care.

Although there have been studies on the use of mobile solutions to manage chronic diseases, there have not been any specific study that primarily focused on researching the potential of mHealth solution to manage diabetes emergencies in Nigeria. Therefore the aim of this paper was to design and implement a simple Tele-health care monitoring system just to monitor and help people with diabetes regardless of their location whenever they develop diabetes emergencies.

Other aspects of the paper are organized as: the proposed system model including hardware system architecture and software design. Finally evaluation was done and conclusion.

\section{Related Works}

Today, there are plethora of diabetes-related applications available in the Apple App Store (iOS operating system; Apple Inc.) and Google Play Store (Android 
operating system; Google), reflecting a rapidly increasing market that may potentially transform the traditional healthcare scheme, providing a large number of useful tools for patients and healthcare providers [10] [11]. Many applications for Android and Apple devices have been developed to manage different aspects of diabetes mellitus.

Intelligent Diabetes Management (IDM): a smartphone application for patients with type 1 diabetes developed by the University of Alberta and launched in 2014. This application includes a glucose and meal tracker, providing a detailed record in a diary format. This application also provides a summary snapshot of the median values of blood glucose from meal to meal and overnight [12].

Glucose Buddy: is another diabetes-related app that allows the users to manually enter their blood glucose readings along with notes and details about their meals. In addition, users may also enter their carbohydrate consumption, insulin dosage, and physical activity. Using the graph function, users may easily track their blood glucose levels over a selected time period [13]. This app also allows users to detect glucose trends, using convenient and user-friendly graphs showing data for 7,14 , or 30 days.

Diabetes Manager: is an all-in-one app that includes an insulin calculator, a carbohydrate database, a favourite's database, and a diary. It is developed for patients with type 1 diabetes mellitus (T1DM) mainly to assist them with the premeal insulin dose calculation [14].

Diabetes Diary: is a self-management tool for patients with diabetes, developed by the Norwegian Centre for e-health Research (NSE) in Tromsø, Norway. It was designed to enable the patients to record blood glucose, insulin, carbohydrates, and physical activity [15].

Diabeo app: is an app used on a mobile phone with an Android or iOS operating system, or on a web portal accessed through any web browser. It has been designed for adults with type 1 or type 2 diabetes on insulin therapy, allowing the patient to record blood glucose levels, insulin doses, physical activity, carbohydrate intake, and hypoglycaemia. It serves as a self-management tool, processing data and calculating bolus insulin dosages according to carbohydrate intake [14] [15].

Diabetes Pal: is an app designed for patients with type 2 diabetes and it is available for both Android and iOS mobile devices. It enables the user to either monitor their blood glucose measurements directly from their glucose meter via Bluetooth or manually by inserting the glucose measurements into the app. The app also enables healthcare professionals to track the patients' glucose measurements, thus serving as a telehealth tool. It also provides text instructions to counter hypoglycemic events [14] [15].

BlueStar: is an in-app diabetes coach available for Android and iOS devices. This was the first mobile app in the USA to be given FDA approval as a mobile prescription therapy [15] [16]. In the BlueStar app, patients can enter their blood glucose levels (wirelessly or manually) and receive real-time coaching. They can 
also organize their medication plan and get advice on their lifestyle and diet. Variables such as blood pressure and body weight are also integrated.

In the work by Lee Gatton et al. in 2010 [17], the mobile application that was developed helps patient with diabetes to classify blood glucose level as either normal, abnormal or emergency, with suggestion on actions to take based on the blood glucose levels. According to Lee, Gatton and Lee, the emergency cases are encouraged to present to hospital as quickly as possible for prompt medical interventions.

In 2012, Salameh [18] developed a mobile phone SMS-based system for diabetes self-management. The system used SMS as a mode of communication between diabetic patients and doctors. The patients sent their blood sugar levels, insulin intake and other data to the doctors, and diabetic patients received feedback in form of motivation messages, warning and instructions via the same medium.

Another app for diabetes management is called "MySugar Logbook". It is a friendly app for patients with diabetes to track their blood glucose throughout the day. It encourages patient engagement through gamification mechanics, activities and rewards, and by using a "monster friend" to represent their progress [19]. MySugar Logbook benefits from multiple integration features, such as Apple Health, glucose meters, and the ability to sync data for export. The app is focused primarily on patients using insulin for diabetes management.

Glooko is a pay-to-use app for Android devices that is said to have a good reputation with users. It is designed to help people see how their diabetes impacts them, personally. It can display a number of key bits of information, including: times of day blood sugar spikes or falls, days of the week diabetes has the biggest impact, how the user's blood sugar levels compare to other data. The built-in food database automatically tracks carbs, and graphs and trends can be viewed in multiple ways [20].

However, a review of the related works revealed the followings about the most of the existing mobile application for the management of diabetic patients;

Its lack use in emergencies, for instance in DKA, HHS or hypoglycaemia.

$>$ Its lack features that make a third party involve in the management of diabetic patients.

In view of the aforementioned drawbacks, this paper presents a system that addresses the aforementioned drawbacks.

\section{Research Methodology}

This section consists of methods and approach used in this work which includes; research approach, system flowchart, implementation and evaluation of the developed system.

\subsection{Research Approach}

Practical and theoretical approach was used. User Centred Design (UCD) was adopted in this work. At the first stage, knowledge was elicited from health care 
providers and literatures on the management of diabetic patients. Multiple views of medical professionals especially diabetes specialists were sought during oneon-one interview, to acquire elaborate knowledge on the management of diabetic patients to enrich the knowledge based of the system.

The followings set of professionals were interviewed; Diabetes specialist doctors, and Diabetes treating nurses. During this stage, diabetic patients and their relatives were interviewed to get firsthand information on the current challenges they were facing in the management of diabetes mellitus especially when patients developed diabetic emergencies. The inputs from both medical professionals, diabetic patients and their relatives inform the features and functionalities of the developed system.

\subsection{Architectural Overview of the System}

Tele-healthcare monitoring systems based on GPS and smartphone which work together in unism to affect the monitoring were used. Within this system, there are two main units: 1) The diabetes patient health monitoring unit, and the 2) Control unit. The first unit consists of biomedical sensors such as blood glucose sensor, GPS receiver and GSM SIM card.

\subsection{GPS Receiver}

The Global Positioning System (GPS) is a space-based global navigation satellite system (GNSS) that provides reliable location and time information in all weather and at all times and anywhere on or near the Earth when and where there is an unobstructed line of sight to four or more GPS satellites [21]. In this paper, GPS receiver can log to Latitude and Longitude of the patient at all time and anywhere. By using the location sent by the GPS periodically, the remote mobile station can receive SMS and understand the position of patient (Latitude and Longitude) and the blood glucose level and hence take an immediate action to intervene and help the patient.

Figure 1 shows the architectural overview of the developed system. The system consists of four (4) units; Patient, Doctor, Patient Next-of-Kin (PNK), and Hospital units. The patient unit consists of the diabetic patient together with smart phone. The mobile application built for the system runs on the mobile phone of the patient. The mobile application contains features and functionalities meant for the management of diabetic patients. Patient and a doctor can communicate either via call or SMS and the data recorded on the mobile application is uploaded automatically to the online storage from time to time.

The doctor unit designates a doctor with mobile phone. The doctor gets either call or SMS from the patient's phone number and responds to him as needs arises. Doctor also has privilege to check previous data of any patient in the online storage for details information about such patient. Such information can be used to guide patients on what to do or what not to do as pertains to management 


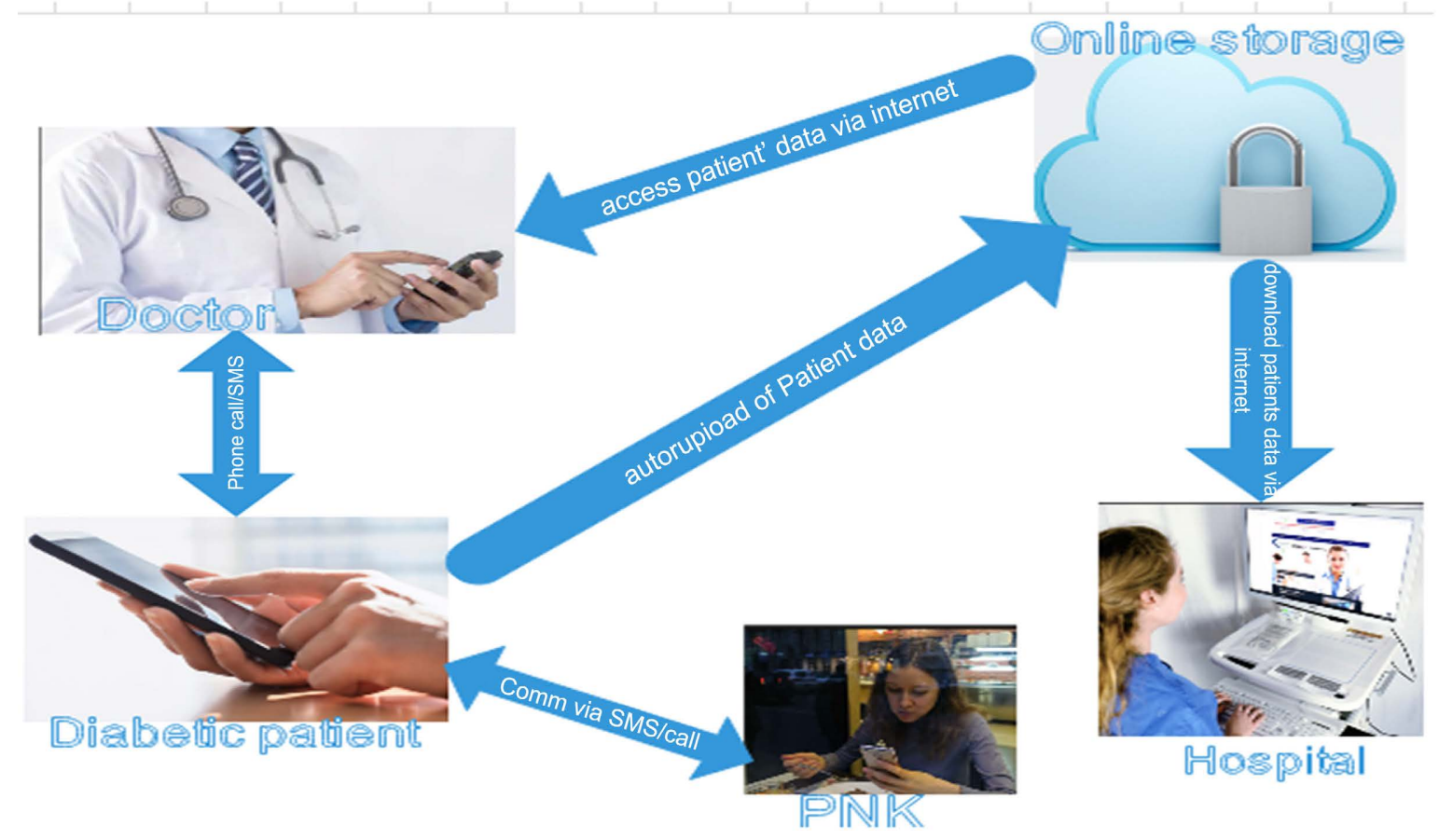

Figure 1. Architectural overview of the system.

of his health condition. Patient-Next-of-Kin was introduced into the design because of the important role they played in the management of chronic disease as diabetes mellitus. The PKN received SMS from patient during critical situations like hyperglycaemic emergencies or hypoglycaemia and such like, and the PKN can communicate via phone call to the patient. The PNK receives SMS from the patient's phone number when the condition of the patient is at critical stage. The hospital unit consists of computer system which can be used for accessing patient's data from online storage. The online storage unit keeps the patient's data for ubiquitous access. With internet connection, doctor and hospital units can access only data during patient visit to such hospital.

This Figure 1 represents the architectural design of the system.

\subsection{System Flowchart and Use Case}

Figure 2 is the flowchart of the system. The flowchart shows all the different conditions and threshold values for different actions that are performed by the system. Upon receiving blood glucose Level (BGL), the mobile application performs any of the functions based on the BGL; analyzes the BGL, initiates a call to a doctor, and sends automatic SMS. If the BGL is not at critical level (that is, not markedly high or too low), the application present functionalities and features for management of both hyperglycemia and hypoglycemia. The final goal of the management is to achieve normal BGL.

\section{Implementation}

The mobile application of the system was developed using Java programming 


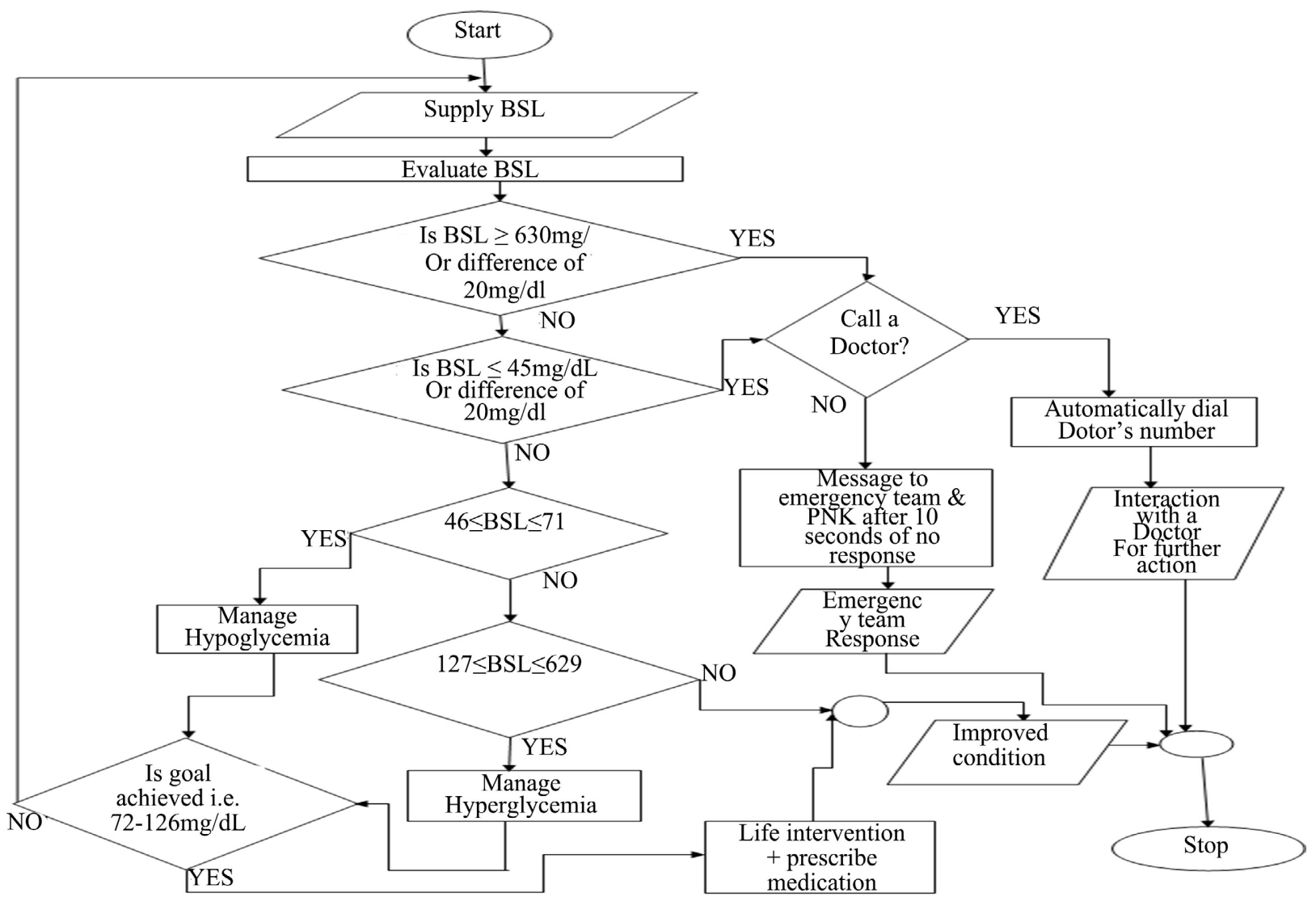

Figure 2. Flowchart for the developed system.

language and it runs on Android operating system. The database of the system was designed using SQLite database. The application runs on Android operating system, and is tested on a mobile smartphone with GPS enabled. The choice of Android OS is because it is open source and non-proprietary, and allows communication and interoperability with other devices.

\section{Evaluation of the Developed System}

The developed system was evaluated using effectiveness and usefulness as metrics.

1) Effectiveness-Effectiveness refers to the extent to which the system behaves in the way that users expect it to and the ease of which users can use it to achieve their specific goals. Equation (1) below was used to evaluate effectiveness of the system.

$$
\% \text { Effectiveness }=\frac{\mathrm{NCC}}{\mathrm{NCC}+\mathrm{NWC}} * \frac{100}{1}
$$

NCC $=$ Number of Correct Calculation

NWC $=$ Number of Wrong Calculation

2) Usefulness-Questionnaires were administered to health care professionals to access the mobile application for the management of diabetes patients and evaluate the application. The response is reported in Section 5.2. 


\section{Result}

In this section, the result obtained from the developed mobile system is captured and reported. The section includes; main features of the system, System's Performance Evaluation, and Users' Perception.

\subsection{Main Features of the System}

In the management of diabetic patients during emergencies, prompt or early intervention required for the management of these conditions is taken into consideration in building this application. The three important personnel that must be taken into consideration in the management of these conditions (patient, doctor, and next-of-kin) and hospital institution are all included as features and functions in the application of this mobile system.

\subsection{Users' Perception}

The following features and functionality of the developed system were examined by health care professionals as a feedback to ascertain the level of acceptability of the system. Table 1 shows the outcome of the survey conducted among potential users of the system. The questionnaires were administered to fifty (50) for evaluations of the system.

Figure 3 is the pie chart, summarizing the output of the survey conducted on

Table 1. Distribution of the respondents' perception of mobile application.

\begin{tabular}{lcc}
\hline \multicolumn{1}{c}{ Item } & Weighted mean score & Rank \\
\hline The application is fast & 4.02 & $1^{\text {st }}$ \\
The application is easy to use & 3.88 & $3^{\text {rd }}$ \\
The application can help reduce the health care cost & 4.00 & $2^{\text {nd }}$ \\
The interface is user friendly & 3.82 & $4^{\text {th }}$ \\
$\begin{array}{l}\text { The application can help in reducing the number of } \\
\text { patient in the hospital }\end{array}$ & 3.50 & $6^{\text {th }}$ \\
Patient's privacy is guaranteed & 3.70 & $5^{\text {th }}$ \\
\hline
\end{tabular}

\section{Frequency}

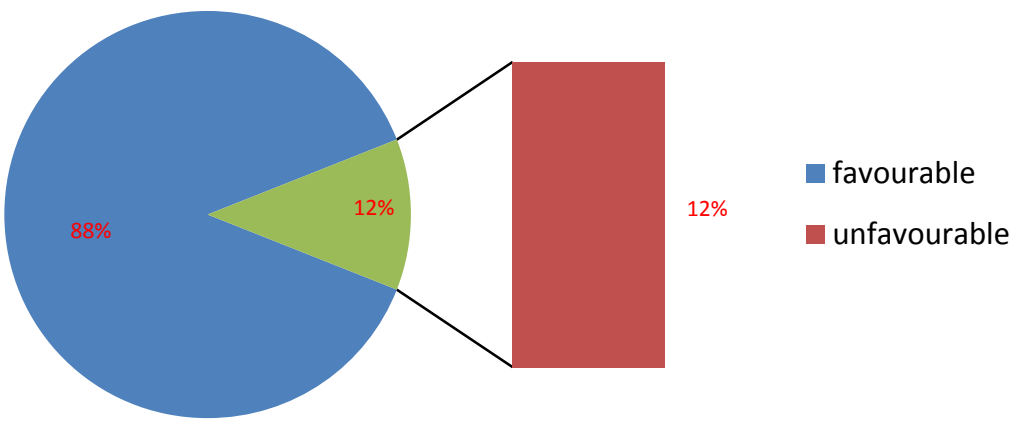

Figure 3. Pie chart of the users' perception of the developed system. 
the users' perception of the developed system. Eighty-eight percent (88\%) of the respondents were favourable to the system.

\section{Conclusions}

In this paper, we introduced a simple and inexpensive telehealth care monitoring system based on a variety of recent wireless technologies such as smart mobile phone, GPS and map website application. The system can effectively help and monitor people with diabetes especially when developed diabetic emergencies when they are far away from healthcare team (managing doctors, family members. etc.). This encourages early management intervention before it becomes too late.

The system's effectiveness is very high in performing its intending purpose and users' perception of the system was highly favourable. This system is capable of reducing mortality rate due to ignorant on the management of diabetes mellitus in developing countries, and from diabetic emergencies as a result of late presentation of patients especially when far away from place of domicile.

\section{Data Availability}

The data to support the findings of this study are included within the article.

\section{Funding}

The authors disclosed no receipt of any financial support for the research, authorship, and/or publication of this article; this work was self sponsored by the authors.

\section{Acknowledgements}

The authors wish to acknowledge all the diabetes specialist physicians and nurses whose inputs and guidance were used during the design of the study protocol and the evaluation of the designed application.

\section{Conflicts of Interest}

The authors declared no potential conflicts of interest with respect to the research to the research, authorship, and/or publication of this article.

\section{References}

[1] World Health Organization (2018). https://www.who.int/news-room/fact-sheets/detail/diabetes

[2] International Diabetes Federation (2019). https://www.idf.org/aboutdiabetes/what-is-diabetes/facts-figures.html

[3] Olamoyegun, M.A., Emuoyibofarhe, O.J., Ala, O.A. and Ugwu, E. (2020) Mobile Phone Use in the Management of Diabetes in Nigeria: A New Potential Weapon. West African Journal of Medicine, 37, 201-208.

[4] Olamoyegun, M.A., Raimi, T.H., Ala, O.A. and Fadare, J.O. (2020) Mobile Phone Ownership to Receive mHealth Services among Patients with Diabetes Mellitus in 
South West, Nigeria. The Pan African Medical Journal, 37, 29.

[5] Adkins, J.W., Storch, E.A., Lewin, A.B., Williams, L., Silverstein, J.H., Malasanos, T. and Geffken, G.R. (2006) Home-Based Behavioral Health Intervention: Use of a Telehealth Model to Address Poor Adherence to Type-1 Diabetes Medical Regimens. Telemedicine Journal and e-Health, 12, 370-372.

https://doi.org/10.1089/tmj.2006.12.370

[6] García-Sáez, G., Hernando, M.E., Martínez-Sarriegui, I., Rigla, M., Torralba, V., Brugués, E., de Leiva, A. and Gómez, E.J. (2009) Architecture of a Wireless Personal Assistant for Telemedical Diabetes Care. International Journal of Medical Informatics, 78, 391-403. https://doi.org/10.1016/j.ijmedinf.2008.12.003

[7] Adeloye, D., Ige, J.O., Aderemi, A.V., et al. (2017) Estimating the Prevalence, Hospitalisation and Mortality from Type 2 Diabetes Mellitus in Nigeria: A Systematic Review and Meta-Analysis. BMJ Open, 7, e015424.

https://doi.org/10.1136/bmjopen-2016-015424

[8] Nkpozi, M.O., Ezeani, I.U., Korubo, I.F., Chinenye, S. and Chapp-Jumbo, A.U. (2019) Outcome of Hyperglycaemic Emergencies in a Tertiary Hospital, South East, Nigeria. Sahel Medical Journal, 22, 47-54.

[9] Wetterhall, S.F., Olson, D.R., DeStefano, F., et al. (1992) Trends in Diabetes and Diabetic Complications, 1980-1987. Diabetes Care, 15, 960-967.

https://doi.org/10.2337/diacare.15.8.960

[10] Whitehead, L. and Seaton, P. (2016) The Effectiveness of Self-Management Mobile Phone and Tablet Apps in Long-Term Condition Management: A Systematic Review. Journal of Medical Internet Research, 18, e97. https://doi.org/10.2196/jmir.4883

[11] Becker, S., Miron-Shatz, T., Schumacher, N., Krocza, J., Diamantidis, C. and Albrecht, U.V. (2014) mHealth 2.0: Experiences, Possibilities, and Perspectives. JMIR mHealth and uHealth, 2, e24. https://doi.org/10.2196/mhealth.3328

[12] Ryan, E.A., et al. (2017) Improved A1C Levels in Type 1 Diabetes with Smartphone App Use. The Canadian Journal of Diabetes, 41, 33-40. https://doi.org/10.1016/j.jcjd.2016.06.001

[13] Aungst, T. (2012) Evaluation of Glucose Buddy App as Diabetes Monitoring Tool for Patients and Clinicians.

https://www.imedicalapps.com/2012/07/glucose-buddy-app-diabetes-patients-clinic $\underline{\text { ians }}$

[14] Ersotelos, N.T., Margioris, A.N., Zhang, X., et al. (2018) Review of Mobile Applications for Optimizing the Follow-Up Care of Patients with Diabetes. Hormones, 17, 541-550. https://doi.org/10.1007/s42000-018-0062-0

[15] Doupis, J., Festas, G., Tsilivigos, C., Efthymiou, V. and Kokkinos, A. (2020) Smartpone-Based Technology in Diabetes Management. Diabetes Therapy, 11, 607-619. https://doi.org/10.1007/s13300-020-00768-3

[16] Agarwal, P., Mukerji, G., Desveaux, L., et al. (2019) Mobile App for Improved Self-Management of Type 2 Diabetes: Multicenter Pragmatic Randomized Controlled Trial. JMIR mHealth and uHealth, 7, e10321. https://doi.org/10.2196/10321

[17] Lee, M., Gatton, T. and Lee, K. (2010) A Monitoring and Advisory System for Diabetes Patient Management Using a Rule-Based Method and KNN. Sensors (Basel), 10, 3934-3953.

[18] Salameh, O. (2012) A Mobile Phone SMS-Based System for Diabetes Self Management. International Arab Journal of e-Technology, 2, 161-166.

[19] Scott, J. (2017) The New MySugr Logbook 3.0 Is Here! MySugar. 
https://mysugr.com/the-new-mysugr-logbook-3-0/

[20] Johnson, J. (2017) Best Diabetes Apps of 2017. Medical News Today. https://www.medicalnewstoday.com/articles/317364.php

[21] Arduino GPS Data to Google Maps GY-NEO6MV2 Part 1, 2015. https://create.arduino.cc/editor/LogM 